\title{
8 \\ Enhancing cognitive capacities over the life-span
}

\author{
Kaarin J Anstey
}

Cognitive capacities refer to memory, thinking, reasoning, problemsolving, planning and processing speed and are also broadly described as aspects of human intelligence. Early psychologists used the term 'intelligence' to refer to what was viewed as a form of 'innate' cognitive ability that was thought to be relatively fixed throughout life and strongly genetically based. However, over the past 50 years, this collection of cognitive abilities has been viewed increasingly in a fluid and dynamic way, recognising that there are strong environmental and experiential influences on cognitive development, as well as genetic influences (Horn 1987).

In order to think about enhancing cognitive function over the lifecourse we must make the assumption that cognitive abilities and their development are not fully predetermined (Baltes 1993). Genetic research has shown that heritability of intelligence increases through adulthood from about 20 per cent in younger adults up to around 80 per cent in old age.

However, this applies to level of cognitive function in old age; the rate of cognitive decline does not seem heritable (Deary 2012). Moreover, studies on heritability have used traditional psychometric 
tests of ability and have not examined more dynamic measures such as neuroplasticity. Variability within individuals may provide scope for improvement and optimising cognitive function (Ackerman 1987).

For several decades it has been recognised that different cognitive abilities appear to be vulnerable to different experiences and neurological conditions (Dixon 2011; Tucker-Drob 2009). Abilities such as reasoning, information processing speed and the higher-level planning and executive function appear to decline more with normal ageing and have been described as 'fluid' abilities or 'mechanic abilities' (Baltes 1987). Abilities involving the accumulation of knowledge and expertise appear to increase slowly through adulthood and are less vulnerable to ageing (Baltes 1987; Horn and Cattell 1967). These have been described as crystallised or pragmatic abilities.

At the societal level, the value of cognitive abilities can be viewed as a form of 'cognitive capital' that enriches a nation's capacity to be innovative and productive, and contributes significantly to wealth. Hence there are both individual- and society-level perspectives to consider in relation to optimising cognitive function.

\section{Why should we aim for optimal cognitive function in an ageing society?}

There are at least three important reasons for research into optimising cognitive development and cognitive health, and the translation of these research findings for public policy. First, as mentioned above, cognitive capital is a resource for society that enables productivity in both the paid and unpaid workforce (Beddington et al. 2008). Greater overall cognitive capital will lead to increased employment and economic benefits. Second, better cognitive function in earlier life reduces the risk of an individual developing cognitive impairment in later life, reduces the risk of dementia and is associated with greater longevity (Deary et al. 2004). Individuals with better cognitive function will take longer to decline to the point where they are impaired. Third, better cognitive function helps individuals age well (Starr et al. 2003). Cognitive function enables many aspects of everyday life, such as managing finances, planning travel, and managing medical conditions. 
Hence, optimising cognitive function will improve quality of life for individuals and societies and reduce the economic burden of healthcare provision as individuals are able to maintain self-integrity and self-care for longer.

\section{Cognitive decline, cognitive impairment and dementia-a continuum}

Importantly, with increasing life expectancy the integrity of the brain and cognition into very old age has become a new focus for research. There is a direct link between cognitive ageing and dementia, with researchers often seeing cognitive function, cognitive impairment and dementia on a continuum (Figure 1), whereas 20 years ago these were often viewed as distinct domains of research. There is much focus on dementia, and the public generally fear developing dementia (Kim et al. 2015; Zeng et al. 2015). However, it is less widely recognised that cognitive impairment that is not severe enough to meet criteria for dementia affects approximately 10 per cent of adults in their $60 \mathrm{~s}$ (Anstey et al. 2013a) and 20 per cent of adults aged 70 and older, about three to four times the number of older adults who have dementia (Plassman et al. 2008). A large proportion of older adults with Mild Cognitive Impairment ultimately develop dementia (Farias et al. 2009) and hence Mild Cognitive Impairment is a strong risk factor for dementia.

Mild Cognitive Impairment impacts on quality of life and the capacity for independent living (Anstey et al. 2013b).

In our epidemiological study, the PATH Through Life, we found that adults aged in their 60s with Mild Cognitive Impairment reported higher rates of difficulties with instrumental activities of daily living such as reading maps, shopping, making telephone calls, and taking medications (Anstey 2013b). Hence, with an ageing population, it is crucial that we identify and implement methods to enable individuals to enhance their cognitive abilities and maintain them into very old age. Ideally, a minimal number of adults will experience cognitive impairment in their old age and few will develop dementia. 


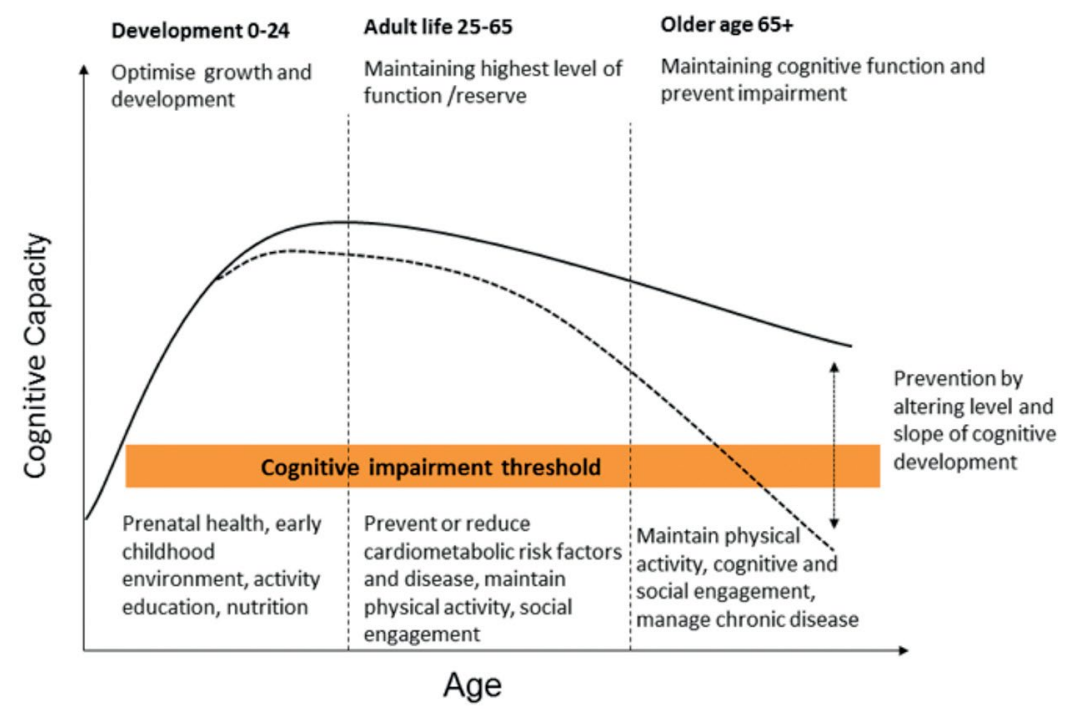

Figure 1. Schematic representation of the trajectory of cognitive development over the life-course

Capacity may vary according to lifestyle, environment, education and health.

Source: Provided by author.

\section{Life-course perspective}

When ageing research focuses exclusively on older adults, for example analysing data from studies of adults aged over 70 years, the contributions of earlier life are often overlooked. In the case of cognitive ageing, the starting point for an individual's cognitive development is prenatal, but the critical point for understanding their trajectory is the peak of cognitive ability reached in the mid-20s and then the rate of decline in cognitive abilities that commences in latemiddle age. It is widely recognised that a life-course perspective needs to be taken into account for the development of cognitive abilities over the life-course (Baltes et al. 1999). Cognitive function in late life is influenced by the accumulation of the impacts of risk factors, insults to the brain, neuropathology, and cognitively enhancing activities that may provide some buffer against the impact of risk factors. 
Figure 1 presents a schematic representation of cognitive development over the life-course, showing that cognitive impairment is on a continuum with normal age-related cognitive decline. The schematic illustrates the trajectory for cognitive growth, showing the peak in the mid-20s for fluid abilities, and the general stability of cognitive function in middle-age, followed by decline in late life. It is clear from this figure that increasing the peak of cognitive function in young adulthood will improve cognitive function for the remainder of the adult life-course. And hence, optimal cognitive ageing requires investment in early cognitive development (Anstey 2014). Similarly, maintaining cognitive function in middle-age will contribute to adults ageing well, minimising risk of cognitive impairment. The fact that cognitive function in late life is the accumulation of both cognitive growth in childhood and early adulthood, and the degree of maintenance through middle-age, means that a life-course approach needs to be taken to cognitive ageing at a public policy and health promotion level.

Taking a life-course approach to optimal cognitive ageing requires focusing different elements of the cognitive trajectories over the lifecourse and developing appropriate interventions for different ages or phases. Typically, cognitive function is stable in middle-age apart from the slowing of reaction time and processing speed. However, by the mid-60s, decline in cognitive function may start to occur. The age at which cognitive abilities start to decline and the rate of decline are influenced by a range of risk and protective factors. An example of how both level and slope of cognitive function may be associated with specific risk factors is shown in Figure 2. This depicts the adult lifecourse cognitive data from the PATH Through Life project for smokers and non-smokers. Smokers perform more poorly at each age, and, in the oldest cohort, are starting to show a faster rate of decline in processing speed than non-smokers. 


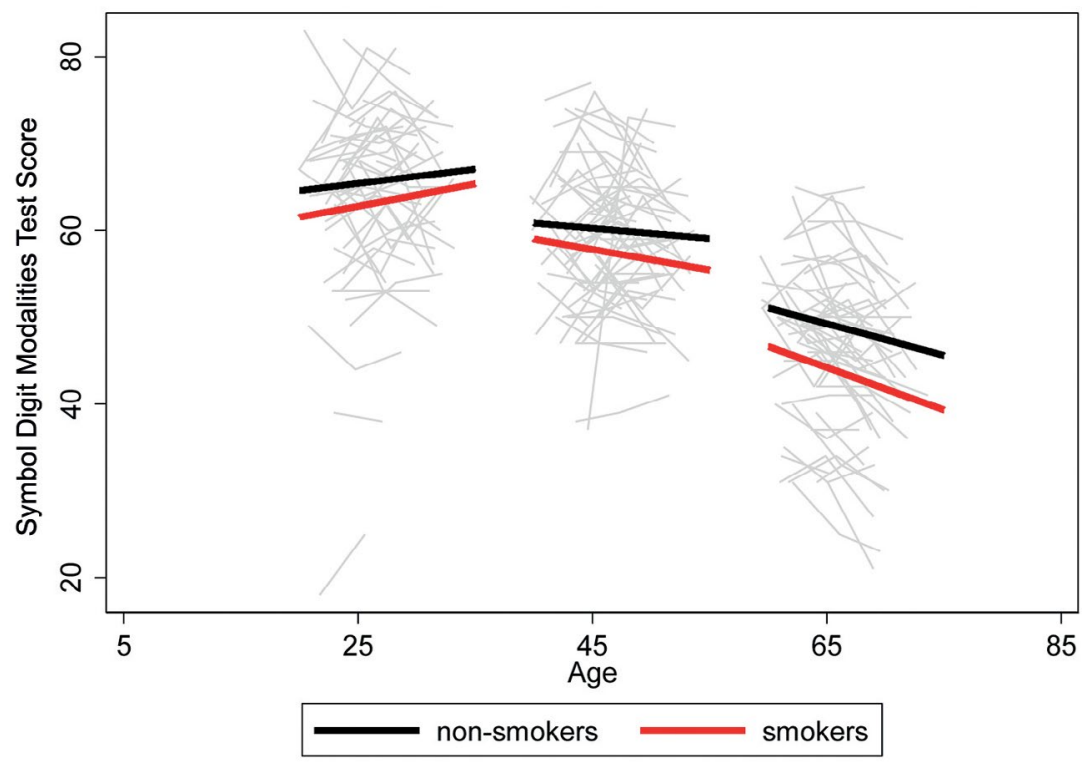

Figure 2. Processing speed ability for smokers and non-smokers aged in their 60s over eight years

Smokers show lower level and faster decline.

Source: Provided by author.

\section{Cognitive reserve}

The 'building of intelligence' in early life through enriched environments and education that promote peak functioning is related to another key concept described by psychologists as 'cognitive reserve' (Stern 2009). This 'reserve' provides individuals with a buffer to sustain their cognitive function when they develop neuropathology or experience diseases or stressors that impact on the brain and subsequent cognitive capacity. The idea of cognitive reserve was introduced originally to account for the finding at autopsy that some individuals who presented with Alzheimer's pathology did not have dementia. In a well-known study of 137 nursing home residents of whom 79 per cent were demented prior to death, 55 per cent had Alzheimer's pathology, 11 per cent had other neuropathology and 11 per cent had no neuropathology (Katzman et al. 1988). Of patients with dementia, 9 per cent had no neuropathology. This study demonstrated that there is no direct correlation between 
neuropathology and dementia and such results have been replicated numerous times over the past two decades. Those without dementia, but who had neuropathology associated with Alzheimer's disease, had more neurones and intact pyramidal neurones and heavier brains than controls. It was therefore postulated that some individuals have a type of 'reserve' capacity, originally described as 'brain reserve' (Richards and Deary 2005; Stern 2002, 2006).

More recent research has used functional Magnetic Resonance Imaging (MRI) to explore the idea of brain and cognitive reserve (e.g. Stern et al. 2005; Stern et al. 2008). Greater activation of specific brain regions during a working memory task has been associated with higher levels of education (Sandry and Sumowski 2014; Sumowski et al. 2014). The idea of cognitive reserve developed from this research to explain the fact that individuals with higher levels of education and higher IQ appear to be at lower risk of dementia and more resilient to the impact of neuropathology on cognitive function. At present, we do not know the full potential of the human brain in terms of how much we can intervene to build cognitive reserve. It is likely that cognitive reserve can be enhanced by cognitive and physical training throughout adulthood.

\section{Neuroplasticity}

Neuroplasticity is related to cognitive reserve and is another key concept for understanding how we may optimise cognitive function (Petrosini et al. 2009; Whalley et al. 2004). Neuroplasticity refers to the capacity of cerebral neural pathways to grow and adapt to environmental exposures, behavioural changes, and specific cognitive and brain training (May 2011). This means that intellectually stimulating activities may maintain and even improve brain function during adulthood, potentially contributing to cognitive reserve and reducing the risk of dementia. Support for the benefit of a cognitively engaged lifestyle has been seen in studies linking lifestyle cognitive activity to the reduced risk of dementia (Fratiglioni et al. 2004). Results from the largest cognitive training study, the ACTIVE trial, have also shown benefits of an intervention that trained older adults in either memory, processing speed or reasoning skills as well as everyday function (Willis et al. 2006). Importantly, long-term 
follow-up of participants trained on reasoning or processing speed showed cognitive benefits were maintained after 10 years (Rebok et al. 2014). Adults who participated in speed of processing training were also less likely to have given up driving over the follow-up period (Edwards et al. 2009).

In another cognitive training study, middle-aged and older adults used the speed of processing training paradigm from the ACTIVE trial and benefits were found for speed of processing training conducted both at home and by groups in a laboratory (Wolinsky et al. 2013). The benefits translated into between two and five years of prevented cognitive decline on standardised cognitive tests. Most cognitive training research has focused on older adults and children with attention deficit disorders; results typically demonstrate that cortical thickness can be increased through intensive repetitive cognitive exercises (Kueider et al. 2012). Research into music training has also demonstrated cognitive and brain benefits in children, but the long-term impact of musical training in childhood is not yet known (Lappe et al. 2011). Research in older adults has shown that there are brain changes associated with memory training (Engvig et al. 2010).

Brain training research in general has been criticised as improvements are only noted on those cognitive capacities focused on in the training; the transfer of benefit to everyday function has rarely been demonstrated. A number of empirical issues relating to dosage, frequency, intensity and type of training have not yet been resolved. Nevertheless, the field is part of a broader movement that will lead to discoveries about how to optimise cognitive function in ways that potentially will persist into late life.

\section{Multidomain influences on cognitive development require a multidisciplinary approach - the CHELM Model}

Influences on cognitive function draw from several different domains, including genetics, environment, sociodemographic factors, health behaviour, and disease (Fotuhi et al. 2012). Developments in education, neuroscience, medicine and psychology have each contributed to an emerging framework for understanding cognitive abilities over the life- 
course. These also speak to potential areas where interventions may be conducted to reduce risk of cognitive decline, and even promote or improve cognitive abilities.

Interventions to address the influences on cognitive development may occur at multiple levels, from changing individual behaviour to changing public policy relating to education, health care or the environment (Fratiglioni and Qiu 2011). Ultimately, the better our society facilitates optimal cognitive development and maintenance, the better we will age as individuals and as a society (Beddington et al. 2008).

A framework for optimising cognitive function is required to pull together the multidomain nature of impacts on cognitive function that will ultimately lead to the level of cognitive ability an individual has in old age (Anstey 2014). I have previously proposed the Cognitive Health Environment Lifestyle Model to depict a framework for understanding influences on cognitive function that may guide policy and intervention.

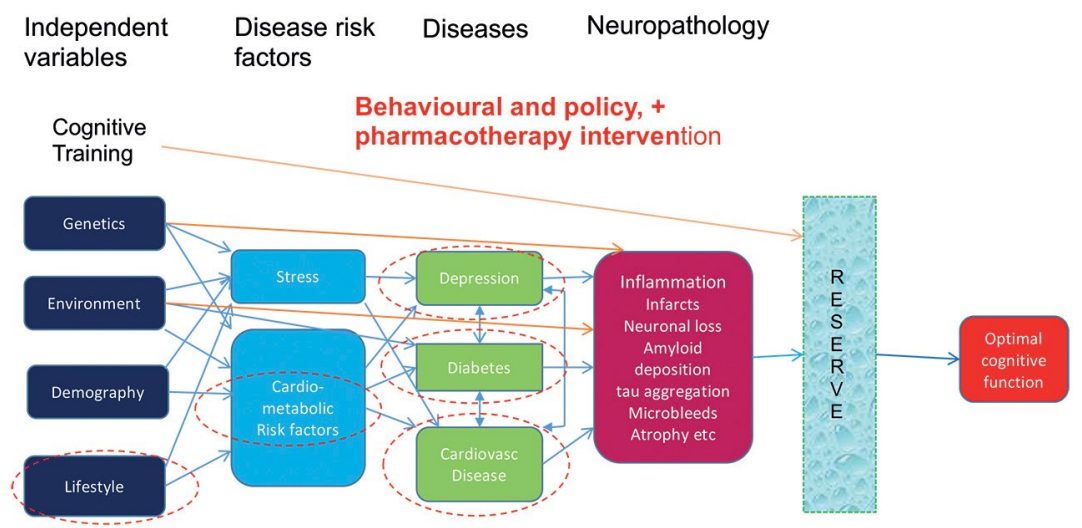

Figure 3. The Cognitive Health Environment and Life Course Model Source: Adapted from Anstey (2014).

This model depicts how various domains influence cognitive outcomes, providing a framework for interventions at the levels of public policy, lifestyle and behavioural changes, and pharmacotherapy.

According to the CHELM framework, intervention can occur through public policy, non-pharmacological behaviour change interventions, pharmacological interventions and activities to develop cognitive 
reserve. Public policy interventions are required to address environmental and contextual influences on cognitive development and cognitive function. These include ensuring as many children as possible receive high-quality education to develop brain structure and brain reserve. Retaining children in school and promoting lifelong learning and occupational training can be influenced by public policy in relation to the provision of education and the maintaining of standards of education.

Public policy is also important for addressing environmental influences such as air pollution in large cities, and the impact of the built environment on the lifestyle behaviours that ultimately impact on the brain and cognition. Environmental factors influencing cognition may, for example, include the design of environments, access to high-quality unprocessed food and access to sporting facilities that encourage individuals to engage in activities that promote health and wellbeing and ward off disease. Establishing building and design codes that take into account the health and capacities of communities and populations with specific needs will ultimately lead to gains in cognitive capital as well as reduced prevalence of chronic diseases. Hence, our society's approach to a broad range of issues and inequality can be viewed in terms of how such factors impact on the cognitive health of individuals, which in turn contribute to how well individuals will age.

Behavioural interventions are required to address risk factors for chronic disease that are also linked to brain and cognitive health. These include sedentary behaviour (Lautenschlager et al. 2010), diet quality (Ashby-Mitchell et al. 2015), smoking (Anstey et al. 2007) and unhealthy alcohol drinking (Anstey et al. 2009). While Body Mass Index (BMI) is not a health behaviour it is a medical condition that may be influenced by health behaviour as well as medical treatment. Both low BMI (underweight) and high BMI (overweight and obese) in middle-age have been linked to increased risk of Alzheimer's disease and dementia (Anstey et al. 2011).

Emerging research in the field of dementia risk reduction is focusing on developing ways of reducing behaviours that increase the risk of cognitive decline and dementia. For example, we have developed an online dementia risk-reduction intervention called Body Brain Life (BBL) (Anstey et al. 2013a). BBL includes seven modules, two of which educate individuals about dementia and dementia risk reduction. Another five modules address individual health behaviours 
using behavioural change principles and techniques such as goalsetting and self-monitoring. Other more intensive interventions to improve cognitive decline have involved walking groups, personal exercise prescription and individual dietary prescription and support (Ngandu et al. 2015; Richard et al. 2012).

Preliminary results from one large European risk-reduction trial has shown that two years post-intervention, cognitive function was improved in the intervention group compared with a control group that received usual care (Ngandu et al. 2015). As depression is an established risk factor for dementia, behavioural interventions to reduce depression are also an avenue for dementia risk reduction. Protective behaviours that reduce risk of dementia include increasing social engagement (Fratiglioni et al. 2004), cognitive engagement (Wilson et al. 2002) and eating fish (Fotuhi et al. 2009).

Pharmaceutical interventions are also appropriate to treat conditions that increase the risk of dementia. These include hypertension, diabetes, heart disease and depression. Recent studies have identified cardiovascular risk factors in young adults influence cognitive ability (Aberg et al. 2009), and cardiovascular risk factors in middle-age influence dementia outcomes in late life (Kivipelto et al. 2001). Other medical conditions linked to cognitive function and decline include head injury and diabetes. However, research is now looking beyond purely medical and health explanations for individual differences in cognitive function.

Environmental factors are of increasing concern in the domain of public health in relation to respiratory function, and there is now evidence for air pollution being associated with poorer cognitive function and Alzheimer's pathology (Calderon-Garciduenas et al. 2012).

There is growing evidence that a range of pesticides and other chemicals are associated with cognitive deficits, yet this area of research is currently limited due to a lack of high-quality data (Zaganas et al. 2013). Environmental factors relating to diet may affect entire populations, in addition to historical effects such as major societal stressors and traumas.

Our society still traditionally views early life as the period during which individuals undertake their education and vocational training. However, research on brain development combined with increasing 
longevity suggests that our perspective on education needs to change. Participation in education at various points during adulthood may have influences on the brain that we have not yet imagined. Bringing all our knowledge together and creating the best possible public policy for cognitive health is likely to promote genuine increases in the numbers of adults who age well, and reduce the prevalence of late life dementia.

\section{Future directions}

Research into factors that reduce cognitive capacity and methods of optimising cognitive capacity is relatively new and many fundamental questions remain unanswered. For example, little is known about how interventions at specific ages may influence longer-term cognitive outcomes, and whether this again differs by sex and genetic risk profile. We still do not know the optimal dosage of most interventions, or for how long they need to be administered. Often cohort studies are limited by the initial measures included in the study, by short follow-up periods or attrition. Intervention studies are typically limited by highly selected and unrepresentative samples, and short follow-up periods, with few interventions lasting as long as two years. In the context of cognitive function over the life-course it is possible that interventions may need to be administered for decades or even lifelong, to demonstrate their potential. Cognitive change in healthy adults is very slow and hence it is difficult to detect the effects of interventions with small samples and over short time-frames.

In addition to unanswered questions about interventions, there are also unanswered questions about the sub-populations in whom they may be trialled. We do not know which interventions may be effective in adults who already have some degree of cognitive impairment and the extent of neural plasticity possible in adults with dementia. Many of our hypotheses relating to optimising cognitive function are based on observational research that links risk and protective factors to better cognition. However, to date there has not been enough research to confirm whether risk modification will lead to reversibility of cognitive decline or improvement. With increasing longevity, it is unknown whether the same risk profiles associated with cognitive decline in say adults in their 80 s will be associated with cognitive 
decline in adults in their 90s or beyond 100. Some recent research has suggested that the increased longevity associated with lack of risk factors (e.g. non-smoking) may actually increase the time spent with cognitive impairment over the entire life-span because adults simply live longer and age is the greatest risk factors for cognitive decline (Anstey et al. 2014). Altogether, there is a need for a major research effort in enhancing cognitive capacities over the adult life-course that will have major benefits for individuals and societies.

\section{Acknowledgements}

This work is funded by NHMRC Fellowship 1002560 and the ARC Centre of Excellence in Population Ageing Research CE110001029.

\section{References}

Aberg Maria, Pedersen Nancy, Toren Kjell, Svartengren Magnus, Backstrand Bjorn, Johnsson Tommy, Cooper-Kuhn Christine, Aberg David, Nilsson Michael and Kuhn George (2009). Cardiovascular fitness is associated with cognition in young adulthood. Proceedings of the National Academy of Sciences of the United States of America, 106(49): 20906-20911.

Ackerman Phillip (1987). Individual differences in skill learning: An integration of psychometric and information processing perspectives. Psychological Bulletin, 120(1): 3-27.

Anstey Kaarin (2014). Optimizing cognitive development over the life course and preventing cognitive decline: Introducing the Cognitive Health Environment Life course Model (CHELM). International Journal of Behavioral Development, 38(1): 1-10.

Anstey Kaarin, Bahar-Fuchs Alex, Herath Pushpani, Rebok George and Cherbuin Nicolas (2013). A 12-week multidomain intervention versus active control to reduce risk of Alzheimer's disease: Study protocol for a randomized controlled trial. Trials, 14: 60. 
Anstey Kaarin, Cherbuin Nicolas, Budge Marc and Young Jennifer (2011). Body mass index in mid life and late-life as a risk factor for dementia: A meta-analysis of prospective studies. Obesity Reviews, 12(5): e426-e437.

Anstey Kaarin, Cherbuin Nicolas, Eramudugolla Ranmalee, SargentCox Kerry, Easteal Simon, Kumar Rajeev and Sachdev Perminder (2013b). Characterizing mild cognitive disorders in the young-old over 8 years: Prevalence, estimated incidence, stability of diagnosis, and impact on IADLs. Alzheimer's and Dementia, 9(6): 640-648.

Anstey Kaarin, Kingston Andrew, Kiely Kim, Luszcz Mary, Mitchell Paul and Jagger Carol (2014). The influence of smoking, sedentary lifestyle and obesity on cognitive impairment-free life expectancy. International Journal of Epidemiology, 43(6): 1874-1883. doi: 10. 1093/ije/dyul 70.

Anstey Kaarin, Mack Holly and Cherbuin Nicolas (2009). Alcohol consumption as a risk factor for dementia and cognitive decline: Meta-analysis of prospective studies. American Journal of Geriatric Psychiatry, 17(7): 542-555.

Anstey Kaarin, von Sanden Chwee, Salim Agus and O'Kearney Richard (2007). Smoking as a risk factor for dementia and cognitive decline: A meta-analysis of prospective studies. The American Journal of Epidemiology, 166(4): 367-378.

Ashby-Mitchell Kimberly, Peeters Anna and Anstey Kaarin (2015). Role of dietary pattern analysis in determining cognitive status in elderly Australian adults. Nutrients, 7(2): 1052-1067.

Baltes Paul (1987). Theoretical propositions of life-span developmental psychology: On the dynamics between growth and decline. Developmental Psychology, 23(5): 611-626.

Baltes Paul (1993). The aging mind: Potential and limits. The Gerontologist, 33(5): 580-594.

Baltes Paul, Staudinger Ursula and Lindenberger Ulman (1999). Lifespan psychology: Theory and application to intellectual functioning. Annual Review of Psychology, 50: 471-507. 
Beddington John, Cooper Cary, Field John, Goswami Usha, Huppert Felicia, Jenkins Rachel, Jones Hannah, Kirkwood Tom, Sahakian Barbara and Thomas Sandy (2008). The mental wealth of nations. Nature, 455(7216): 1057-1060.

Calderon-Garciduenas Lillian, Kavanaugh Michael, Block Michelle, D'Angiulli Amedeo, Delgado-Chavez Ricardo, Torres-Jardon Ricardo, Gonzalas-Maciel Angelica, Reynoso-Robles Rafael, Osnaya Norma, Villareal-Calderon, Guo Ruixin, Hua Zhoawei, Zhu Honghi, Perry George and Diaz Phillipe (2012). Neuroinflammation, hyperphosphorylated tau, diffuse amyloid plaques, and down-regulation of the cellular prion protein in air pollution exposed children and young adults. Journal of Alzheimer's disease, 28(1): 93-107.

Deary Ian (2012). Intelligence. Annual Review of Psychology, 63: 453-482.

Deary Ian, Whiteman Martha, Starr John, Whalley Lawrence and Fox Helen (2004). The impact of childhood intelligence on later life: Following up the Scottish mental surveys of 1932 and 1947. Journal of Personality and Social Psychology, 86(1): 130-147.

Dixon Roger (2011). Enduring theoretical themes in psychological aging: Derivation, functions, perspectives, and opportunities. In KW Schaie and SL Willis (Eds) Handbook of the Psychology of Aging (7th edition). San Diego: Academic Press, pp. 3-23.

Edwards Jerri, Myers Charlsie, Ross Lesley, Roenker Daniel, Cissell Gayla, McLaughlin Alexis and Ball Karlene (2009). The longitudinal impact of cognitive speed of processing training on driving mobility. The Gerontologist, 49(4): 485-494.

Engvig Andreas, Fjell Anders, Westlye Lars, Moberget Torgeir, Sundseth Oyvind, Larsen Vivi and Walhovd Kristine (2010). Effects of memory training on cortical thickness in the elderly. NeuroImage, 52(4): 1667-1676.

Farias Sarah, Mungas Dan, Reed Bruce, Harvey Danielle and DeCarli Charles (2009). Progression of mild cognitive impairment to dementia in clinic- vs community-based cohorts. Archives of Neurology, 66(9): 1151-1157. 
Fotuhi Majid, Do David and Jack Clifford (2012). Modifiable factors that alter the size of the hippocampus with ageing. Nature Reviews Neurology, 8(4): 189-202.

Fotuhi Majid, Mohassel Payam and Yaffe Kristine (2009). Fish consumption, long-chain omega-3 fatty acids and risk of cognitive decline or Alzheimer disease: A complex association. Nature Clinical Practice Neurology, 5(3): 140-152.

Fratiglioni Laura, Paillard-Borg Stephanie and Winblad Bengt (2004). An active and socially integrated lifestyle in late life might protect against dementia. The Lancet Neurology, 3(6): 343-353.

Fratiglioni Laura and Qiu Chengxuan (2011). Prevention of cognitive decline in ageing: Dementia as the target, delayed onset as the goal [Comment]. The Lancet Neurology, 10(9): 778-779.

Horn John (1987). A context for understanding information processing studies of human abilities. In PA Vernon (Ed) Speed of information processing and intelligence. Norwood, NJ: Ablex, pp. 201-238.

Horn John and Cattell Raymond (1967). Age differences in fluid and crystallized intelligence. Acta Psychologica, 26(2): 107-129.

Katzman Robert, Terry Robert, DeTeresa Richard, Brown Theodore, Davies Peter, Fuld Paula, Renbing Xiong and Peck Arthur (1988). Clinical, pathological, and neurochemical changes in dementia: A subgroup with preserved mental status and numerous neocortical plaques. Ann Neurol, 23(2): 138-144.

Kim Sarang, Sargent-Cox Kerry and Anstey Kaarin (2015). A qualitative study of older and middle-aged adults' perception and attitudes towards dementia and dementia risk reduction. Journal of Advanced Nursing, 71(7): 1694-1703. doi: 10.1111/jan.12641.

Kivipelto Miia, Helkala Eeva, Laakso Mikko, Hanninen Tuomo, Hallikainen Merja, Alhainen Kari, Soininen Hilkka, Tuomilehto Jaakko and Nissinen Aulikki (2001). Midlife vascular risk factors and Alzheimer's disease in later life: Longitudinal, population based study. The British Medical Journal, 322(7300): 1447-1451. 
Kueider Alexandra, Parisi Jeanine, Gross Alden and Rebok George (2012). Computerized cognitive training with older adults: A systematic review. PLoS One, 7(7): e40588.

Lappe Claudia, Trainor Laurel, Herholz Sibylle and Pantev Christo (2011). Cortical plasticity induced by short-term multimodal musical rhythm training. PLoS One, 6(6): e21493.

Lautenschlager Nicola, Cox Kay and Kurz Alexander (2010). Physical activity and mild cognitive impairment and Alzheimer's disease. Current Neurology and Neuroscience Reports, 10(5): 352-358.

May Arne (2011). Experience-dependent structural plasticity in the adult human brain. Trends in Cognitive Sciences, 15(10): 475-482.

Ngandu Tiia, Lehtisalo Jenni, Solomon Alina, Levalahti Esko, Ahtiluoto Satu, Antikainen Riitta, Bäckman Lars, Hänninen Tuomo, Jula Antti, Laatikainen Tiina, Lindström Jaana, Mangialasche Francesca, Paajanen Teemu, Pajala Satu, Peltonen Markku, Rauramaa Rainer, Stigsdotter-Neely Anna, Strandberg Timo, Tuomilehto Jaako, Soininen Hikka and Kivipelto Miia (2015). A 2-year multidomain intervention of diet, exercise, cognitive training, and vascular risk monitoring versus control to prevent cognitive decline in at-risk elderly people (FINGER): A randomised controlled trial. The Lancet, 385(9984): 2255-2263.

Petrosini Laura, De Bartolo Paola, Foti Francesca, Gelfo Francessca, Cutuli Debora, Leggio Maria and Mandolesi Laura (2009). On whether the environmental enrichment may provide cognitive and brain reserves. Brain Research Reviews, 61(2): 221-239.

Plassman Brenda, Langa Kenneth, Fisher Gwenith, Heeringa Stephen, Weir David, Ofstedal Mary, Burke James, Hurd Michael, Potter Guy, Rodgers Willard, Steffens David, McArdle John and Wallace Robert (2008). Prevalence of cognitive impairment without dementia in the United States. Annals Internal Medicine, 148(6): 427-434.

Rebok George, Ball Karlene, Guey Lin, Jones Richard, Kim HaeYoung, King Jonathan, Willis Sherry (2014). Ten-Year Effects of the advanced cognitive training for independent and vital elderly cognitive training trial on cognition and everyday functioning in older adults. Journal of the American Geriatrics Society, 62(1): 16-24. 
Richard Edo, Andrieu Sandrine, Solomon Alina, Mangialasche Francesca, Ahtiluoto Satu, Moll van Charante Eric, Coley Nicola, Fratiglioni Laura, Neeley Anna Stigsditter, Vellas Bruno, van Gool Willem and Kivipelto Miia (2012). Methodological challenges in designing dementia prevention trials - the European Dementia Prevention Initiative (EDPI). Journal of the Neurological Sciences, 322(1-2): 64-70.

Richards Marcus and Deary Ian (2005). A life course approach to cognitive reserve: A model for cognitive aging and development? Annals of Neurology, 58(4): 617-622.

Sandry Joshua and Sumowski James (2014). Working memory mediates the relationship between intellectual enrichment and long-term memory in multiple sclerosis: An exploratory analysis of cognitive reserve. Journal of the International Neuropsychological Society, 20(8): 868-872.

Starr John, Deary Ian and Macintyre Sally (2003). Associations with successful ageing in the 'Healthy old people in Edinburgh' cohort: Being well, fit and healthy. Aging Clinical and Experimental Research, 15(4): 336-342.

Stern Yaakov (2002). What is cognitive reserve? Theory and research application of the reserve concept. Journal of the International Neuropsychological Society, 8(3): 448-460.

Stern Yaakov (2006). Cognitive reserve and Alzheimer disease. Alzheimer Disease and Associated Disorders, 20: 112-117.

Stern Yaakov (2009). Cognitive reserve. Neuropsychologia, 47(10): 2015-2028.

Stern Yaakov, Habeck Christian, Moeller James, Scarmeas Nikolaos, Anderson Karen, Hilton John, van Heertum Ronald (2005). Brain networks associated with cognitive reserve in healthy young and old adults. Cerebral Cortex, 15(4): 394-402. doi: 10.1093/cercor/bhh142.

Stern Yaakov, Zarahn Eric, Habeck Christian, Holtzer Roee, Rakitin Brian, Kumar Arjun, Flynn Josephm Steffener Jaron and Brown Truman (2008). A common neural network for cognitive reserve in verbal and object working memory in young but not old. Cerebral Cortex, 18(4): 959-967. 
Sumowski James, Rocca Maria, Leavitt Victoria, Dackovic Jelena, Mesaros Sarlota, Drulovic Jelena, DeLuca John and Filippi Massimo (2014). Brain reserve and cognitive reserve protect against cognitive decline over 4.5 years in MS. Neurology, 82(20): 1776-1783.

Tucker-Drob Elliot (2009). Differentiation of cognitive abilities across the life span. Developmental Psychology, 45(4): 1097-1118.

Whalley Lawrence J, Deary Ian, Appleton Charlotte and Starr John (2004). Cognitive reserve and the neurobiology of cognitive aging. Ageing Research Reviews, 3(4): 369-382.

Willis Sherry, Tennstedt Sharon, Marsiske Michael, Ball Karlene, Elias Jeffrey, Koepke Kathy, Morris John, Rebok George, Unverzagt Frederick, Stoddard Anne and Wright Elizabeth (2006). Longterm effects of cognitive training on everyday functional outcomes in older adults. The Journal of the American Medical Assocation, 296(23): 2805-2814.

Wilson Robert, Mendes de Leon Carlos, Barnes Lisa, Schneider Julie, Bienias Julia, Evans Denis and Bennett David (2002). Participation in cognitively stimulating activities and risk of incident Alzheimer disease. The Journal of the American Medical Assocation, 287(6): $742-748$.

Wolinsky Fredric, Vander Weg Mark, Howren Bryant, Jones Michael and Dotson Megan (2013). A randomized controlled trial of cognitive training using a visual speed of processing intervention in middle aged and older adults. PLoS One, 8(5) e61624.

Zaganas Ioannis, Kapetanaki Stefania, Mastorodemos Vassileios, Kanavouras Konstantinos, Colosio Claudio, Wilks Martin and Tsatsakis Aristidis (2013). Linking pesticide exposure and dementia: What is the evidence? Toxicology, 307: 3-11.

Zeng Fan, Xie Wan-Ting, Wang Yan-Jiang, Luo Hong-Bo, Shi XiangQun, Zou Hai-Qiang, Zeng Yue-Ring, Li Ya-Fei, Zhang Shao-Rong and Lian Yan (2015). General public perceptions and attitudes toward Alzheimer's disease from five cities in China. Journal of Alzheimer's Disease, 43(2): 511-518. 
This text is taken from Population Ageing and Australia's Future, edited by Hal Kendig, Peter McDonald and John Piggott, published 2016 by ANU Press, The Australian National University, Canberra, Australia. 